\title{
Simultaneous Observation of Hybrid States for Cyber-Physical Systems: A Case Study of Electric Vehicle Powertrain
}

\author{
Chen Lv, Yahui Liu, Xiaosong Hu, Hongyan Guo, Dongpu Cao, Fei-Yue Wang
}

\begin{abstract}
As a typical cyber-physical system (CPS), electrified vehicle becomes a hot research topic due to its high efficiency and low emissions. In order to develop advanced electric powertrains, accurate estimations of the unmeasurable hybrid states, including discrete backlash nonlinearity and continuous half-shaft torque, are of great importance. In this paper, a novel estimation algorithm for simultaneously identifying the backlash position and half-shaft torque of an electric powertrain is proposed using a hybrid system approach. System models, including the electric powertrain and vehicle dynamics models, are established considering the drivetrain backlash and flexibility, and also calibrated and validated using vehicle road testing data. Based on the developed system models, the powertrain behavior is represented using hybrid automata according to the piecewise affine property of the backlash dynamics. A hybrid-state observer, which is comprised of a discrete-state observer and a continuousstate observer, is designed for the simultaneous estimation of the backlash position and half-shaft torque. In order to guarantee the stability and reachability, the convergence property of the proposed observer is investigated. The proposed observer are validated under highly dynamical transitions of vehicle states. The validation results demonstrates the feasibility and effectiveness of the proposed hybrid-state observer.
\end{abstract}

Index Terms - Simultaneous observation, cyber physical system, hybrid states, backlash nonlinearity, electric powertrain.

\section{INTRODUCTION}

$\mathrm{C}$ YBER physical system, a distributed, networked system that fuses computational processes with the physical world exhibiting a multidisciplinary nature, has recently become a research focus [1-3]. Besides, the ever-heavier burden on the environmental protection and energy conservation requires ground vehicles to be more efficient and cleaner. Thus, as a typical CPS, electrified vehicles (EV), which make mobility greener and more efficient, is gaining increasing attention from academia, automotive industry and governmental organizations [4,5]. Electric vehicle technologies, such as powertrain electrification, high-efficient combustion, automated driving and alternative energy have been widely studied and developed. Among these solutions, various types of electrified powertrain systems show great promises, due to their advantages in highefficiency energy conversion and the decreased or even zero emission [6].

In an electric powertrain, the controller, representing the "cyber" world, the physical plant, the driver, the "human", and the environment, are highly coupled and interacted, determining vehicle's overall performance jointly [7, 8]. These complex subsystems with strong uncertainties, hard nonlinearities and multi-disciplinary interactions make the estimation, control and optimization of electrified powertrains very difficult [9-11]. Therefore, there are still a number of fundamental and critical issues in advanced powertrain control and optimization remained open [12-14].

There are two key factors of hybrid states that greatly influence the control performance of an electrified powertrain: the backlash and the flexibility [15]. Backlash, which is of discrete state, introduces a hard nonlinearity into the powertrain control loop for torque generation and transmission. The main source of backlash is play between gears in the final drive and the gearbox, but other plays throughout the drivetrain add to the backlash. Flexibility is another main excitation source of oscillations on continuous half-shaft torque [16, 17]. It is mainly contributed by the elastic propeller and half-shafts. Hence, when controlling the powertrain, one of the limitations of the control loop is the capability to compensate for backlash and drivetrain flexibility.

In order to develop a high-performance compensatory control system for vehicle powertrain, the hybrid-state information of the discrete backlash position and continuous half-shaft torque, is of great importance [18, 19]. This is particularly essential when the driver requests the electric vehicle to go from acceleration to regenerative braking or vice versa. During the tip-in and tip-out maneuvers, the backlash is firstly traversed, and no torque is transmitted through the shaft during this period. After the backlash is engaged, power transmission is then recovered, but the contact of the gears would lead to a great impact, further exciting the driveline nonlinearity, and causes unexpected torsional oscillations [20]. Therefore, to improve the performance of the electric powertrain, the advanced control must compensate for backlash nonlinearity and powertrain flexibility [21].

In the vehicle onboard applications, the backlash position and half-shaft torque are unable to be directly measured by sensors. 
Besides, in the existing studies, the observations of backlash position and half-shaft torque in automotive powertrain systems are usually treated independently, and little has been reported on simultaneous estimation of these two variables.

In [22], a method for the observation of backlash in gear systems of servomechanisms was proposed based on a momentum transfer analysis. It showed that the change in the speed of the primary gear is related to the magnitude of the backlash. In [23], motivated by applications for automotive powertrains, nonlinear observers for backlash size and state estimation in a rotating system were developed by using eventbased Kalman filters. Simulation results showed that the developed estimator followed the real position during backlash traverse accurately with good robustness, even with huge errors in some system parameters. In [24], scholars dealt with the observation of mechanical backlash in electrical drives. The proposed method utilizes the motor velocity as the only measurement signal, and was processed based on the beginning and the end of the dead zone.

On the other side, in some automotive powertrain studies, scholars have carried out lots of work on torque estimation of flexible haft-shaft without considering the impact of nonlinear backlash. In [25], a low-cost approach for axle shaft torque estimation was proposed using nonlinear sliding mode techniques. It used the signals of the output speed of the transmission and driven wheel speed. In [26], a haft-shaft torque estimator was developed for a class of switching systems with an application in a vehicle transmission system. Model uncertainties were treated as disturbance inputs to the system and the gains of the proposed observer were obtained by convex optimization under large variations of operation condition. In [27], oriented by torque compensatory controller synthesis for a four-wheel drive electric vehicle, a reduced-order observer for half-shaft torque estimation was developed using linear observation approach. Nevertheless, the backlash nonlinearity and flexible property do exist in the automotive powertrain at the same time, but simultaneous estimations of their states have rarely been reported.

This study aims to simultaneously identify the backlash position and half-shaft torque, which are unable to be measured directly in production vehicles, with input available states of vehicle and powertrain using a hybrid system approach. And the proposed observation algorithm can be applied later to active control of powertrain systems for enhancing drivetrain performance and vehicle drivability. The main contribution of this work lies in the following aspects: (1) the powertrain behavior is represented using the hybrid automata based on the piecewise affine characteristics of the backlash nonlinearity; (2) a novel hybrid-state observer, including a discrete-state observer and a continuous one, is proposed for simultaneously estimating the backlash position and half-shaft torque, and its feasibility and effectiveness are successfully validated; (3) the effective estimations of the backlash and half-shaft torque could help to set up a solid foundation for future studies in compensatory controller synthesis for powertrain systems.

The rest of the article is organized as follows. System models, including the electric powertrain and vehicle dynamics models, are established with consideration of drivetrain backlash and flexibility in Section II. The piecewise affined powertrain dynamics model is represented using the hybrid system theory, i.e. the hybrid automata in Section III. In Section IV, the hybrid-state observer is proposed for the simultaneous estimation of the backlash position and half-shaft torque. The convergence property of the proposed hybrid-state observer is investigated in Section V. Observer validation and results analysis are carried out in Section VI, which is followed by the concluding remarks in Section VII.

\section{Dynamical Modelling Of AN Electrified POWERTRAIN}

Fig. 1 shows the overall structure of the electric powertrain system studied in this paper. The electric powertrain is comprised of an electric motor, a gearbox, a final drive, a differential, and two half shafts. The electric motor is installed at the centre of the front axle of the vehicle. During acceleration and cruising processes, the motor provides a propulsion torque, which is transmitted via the drivetrain and exerted on the axle, propelling the vehicle. While in deceleration procedures, the electric motor works in its regenerative brake mode, supplying a braking torque, and decelerates the vehicle. The detailed mathematical models of the electrified powertrain system are described below.

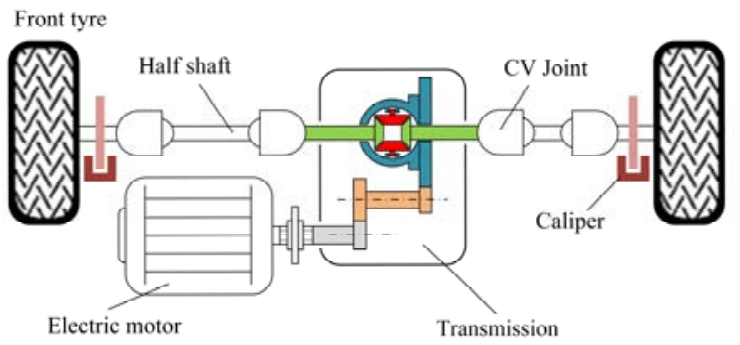

Fig. 1. Overall structure of the electric powertrain system.

\section{A. The Electrified Powertrain Modelling}

According to the physical structure of the plant, a simplified two-inertia model of the electric powertrain is abstracted, as Fig. 2 shows. In this two-inertia model, one inertia represents the electric motor, and the other one indicates the dynamical load. The gearbox, which includes the transmission, final drive, differential, and two constant-velocity $(\mathrm{CV})$ joints, is located at the side of the motor inertia. The nonlinear backlash, which contributions throughout the powertrain and affects the drivetrain dynamics, is described by a backlash angle, noted as $2 \alpha$. The flexible half shaft is modelled with the representation of its stiffness and damping characteristics. And the output torque of the electric motor is considered to be equally delivered to the left and right half shafts.

Considering the dynamics of the electrical system, the model of the motor torque is established as a first-order reaction [28], as equation (1) shows.

$$
\tau_{m} \dot{T}_{m}(t)+T_{m}(t)=T_{m, r e f}(t)
$$

where $T_{m}$ is the actual value of motor torque, $T_{m, \text { ref }}$ is the referenced one, and $\tau_{m}$ is a time constant, which is taken as 0.05 . 


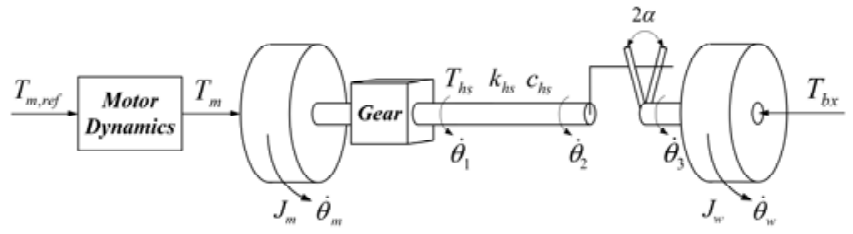

Fig. 2. Simplified two-inertia model of the electric powertrain.

The motor dynamics, which can be seen as the dynamical relationship between the motor's output torque and the halfshaft torque, can be given by:

$$
J_{m} \ddot{\theta}_{m}(t)+b_{m} \dot{\theta}_{m}(t)=T_{m}(t)-2 T_{h s}(t) / i_{0} i_{g}
$$

where, $J_{m}$ is the motor inertia, $b_{m}$ is the viscous frictional index of the electric motor, $i_{0}$ and $i_{g}$ are the gear ratios of the final drive and the transmission system, respectively. $T_{h s}$ indicates the half-shaft torque

A half shaft with a nonlinear backlash gap connects the wheel inertia and the gearbox. The nonlinear flexible half-shaft torque can be expressed by [29]:

$$
\begin{gathered}
T_{h s}(t)=k_{h s} \theta_{s}(t)+c_{h s} \dot{\theta}_{s}(t) \\
\theta_{s}(t)=\theta_{d}(t)-\theta_{b}(t) \\
\theta_{d}(t)=\theta_{1}(t)-\theta_{3}(t), \theta_{b}(t)=\theta_{2}(t)-\theta_{3}(t)
\end{gathered}
$$

where, $k_{h s}$ and $c_{h s}$ represent the stiffness and damping coefficients of the half shaft, separately; $\theta_{d}$ indicates the twist angle of the half shaft; $\theta_{b}$ represents the backlash position; and $\theta_{1}, \theta_{2}$ and $\theta_{3}$ are the angles at the indicated positions on the half shaft, respectively, as Fig. 2 shows, where $\theta_{1}=\theta_{m} / i_{0} i_{g}$ and $\theta_{3}=\theta_{w}$.

The nonlinear backlash model can be described by the following [12]:

$$
\dot{\theta}_{b}(t)= \begin{cases}\max \left(0, \dot{\theta}_{d}(t)+\frac{k_{h s}}{c_{h s}}\left(\theta_{d}(t)-\theta_{b}(t)\right)\right) & , \theta_{b}(t)=-\alpha \\ \dot{\theta}_{d}(t)+\frac{k_{h s}}{c_{h s}}\left(\theta_{d}(t)-\theta_{b}(t)\right) & ,\left|\theta_{b}(t)\right|<\alpha \\ \min \left(0, \dot{\theta}_{d}(t)+\frac{k_{h s}}{c_{h s}}\left(\theta_{d}(t)-\theta_{b}(t)\right)\right) & , \theta_{b}(t)=\alpha\end{cases}
$$

The load dynamics of the electrified powertrain can be given by:

$$
J_{w} \ddot{\theta}_{w}(t)+b_{w} \dot{\theta}_{w}(t)=T_{h s}(t)-T_{h b}(t)-T_{b x}(t)
$$

where, $J_{w}$ represents the wheel inertia, the load contributed by the road is divided into a frictional term $b_{w}$ and the tyre longitudinal force $T_{b x}$, respectively. The frictional resistance torque $T_{h b}$, generated by the hydraulic brakes, can be regarded as a disturbance to the system.

The frictional braking torque $T_{h b}$ can be represented by [30]:

$$
T_{h b}=2 \mu_{b} \pi r_{F W}^{2} r_{b} p_{F W}(t)
$$

where $\mu_{b}$ represents the friction coefficient of the brake disc, $r_{F W}$ is the piston's radius of the front-wheel brake cylinder, and $r_{b}$ is the effective frictional radius of the brake disc. $p_{F W}$ is the brake pressure of the front-wheel cylinder.

Considering the hydraulic flow property of the brake fluid and valve dynamics, the model of the wheel cylinder pressure can be described as [30]:

$$
\dot{p}_{F W}(t)=\frac{k_{F W}}{\pi^{2} r_{F W}^{4}} C_{d} A_{v}(t) \sqrt{\frac{2 \Delta p(t)}{\rho_{f l u}}}
$$

where, $k_{F W}$ is the spring stiffness of the wheel brake cylinder, $\mathrm{Cd}$ and Av are the flow coefficient and cross section area of the valve opening, respectively. $\rho_{f l u}$ is the density of the hydraulic fluid, and $\Delta p$ is the pressure difference across the valve.

In some existing studies of estimation and control of the powertrain system, the wheel slip was usually neglected [31]. However, since the tyre's dynamical behaviour is of great importance for research on vehicle acceleration and deceleration, in order to achieve more accurate results, in this study we adopt the Pacejka magic formula tyre model [32]. The tyre behaviour can be described accurately by a combined model with longitudinal and lateral slip. Besides, the vehicle dynamical model with eight degrees of freedom has also been established in the environment of MATLAB/Simulink. Some key parameters are listed in Table 1. The detailed description of the nonlinear vehicle models have been reported in [30].

TABLE I

Key Parameters of the Vehicle and the Electrified Powertrain

\begin{tabular}{cccc}
\hline \hline & Parameter & Value & Unit \\
\hline \multirow{2}{*}{ Electric motor } & Peak power & 45 & $\mathrm{~kW}$ \\
& Maximum torque & 144 & $\mathrm{Nm}$ \\
\hline \multirow{2}{*}{ Battery } & Voltage & 326 & $\mathrm{~V}$ \\
& Capacity & 66 & $\mathrm{Ah}$ \\
\hline \multirow{5}{*}{ Vehicle } & Total mass & 1360 & $\mathrm{~kg}$ \\
& Wheel base & 2.50 & $\mathrm{~m}$ \\
& Coefficient of air & 0.32 & - \\
& Resistance & 0.295 & $\mathrm{~m}$ \\
& Nominal radius of tyre & 7.881 & - \\
& Gear ratio & $96 \%$ & - \\
\hline \hline
\end{tabular}

B. Experimental Verification of the Powertrain Model

In order to guarantee the feasibility and effectiveness of the established system models, parameter calibration and model verification needs to be carried out before simulation study.

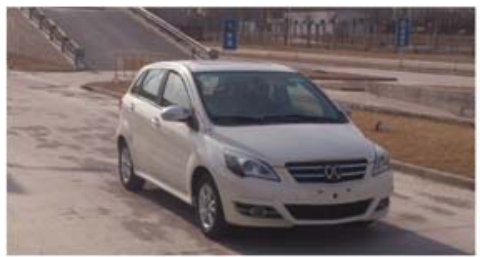

(a)

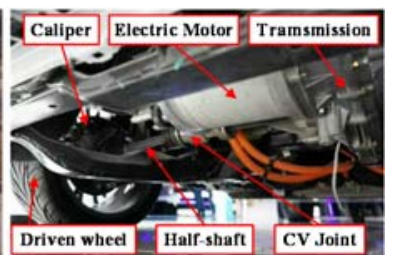

(b)
Fig. 3. Pictures of the test vehicle and the electrified powertrain system.

Firstly, real vehicle road testing is implemented in the test field. Tests of vehicle acceleration, regenerative deceleration and state transitions between driving and braking are carried out. Fig. 3 (a) and (b) show the test vehicle and the electrified powertrain system, respectively. A series of state variables of the powertrain, including the output torque of the electric motor, motor rotational speed, wheel speed and vehicle speed, are measured by sensors and collected during the experiments.

Then, the vehicle and electric powertrain models which has been established in MATLAB/Simulink are fed with the experimental data for parameter calibration. As the first subplot in Fig. 4 shows, the motor's output torque measured in tests is 
used as the input signal of the developed models, and the output signals of those state variables, including motor speed, wheel speed, and vehicle speed, are generated by the models and compared with the real experimental data. According to Fig. 4, the simulation results of the model match with the real experimental data quite well, showing the feasibility and correctness of the developed electric powertrain model.

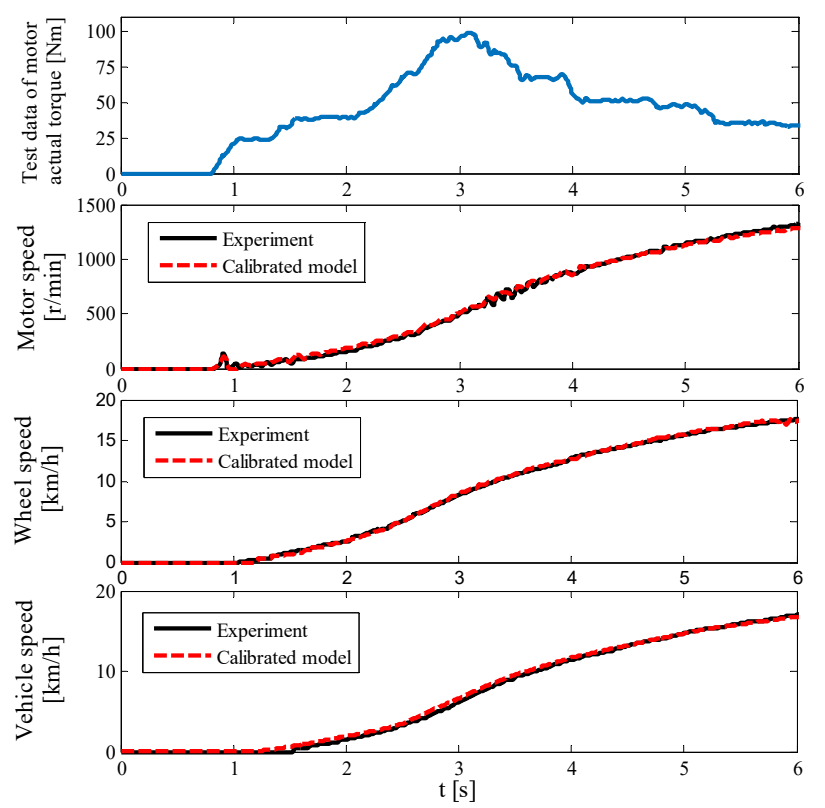

Fig. 4. Validation results of the electrified powertrain model.

\section{HYBRID REPRESENTATION OF THE SYSTEM}

Due to the piecewise affine dynamics of the nonlinear backlash model shown in equation (6), it is suitable to describe the powertrain behavior using hybrid system approach, which is a powerful system modelling methodology [33].

Definition 1. A hybrid system $\mathcal{H}$ is a collection:

$$
\mathcal{H}=(Q, \sigma, \psi, X, U, D, Y, \text { Init, } f, h, R)
$$

where,

- $Q$ denotes the finite set of discrete states of the system;

- $\sigma$ and $\psi$ represent the finite set of discrete input and output variables, respectively;

- $X \subseteq \mathbb{R}^{n}, U \subseteq \mathbb{R}^{p}, D \subseteq \mathbb{R}^{n}$ and $Y \subseteq \mathbb{R}^{p}$ indicate the domains of continuous state, input, disturbance, and output variables, individually;
- Init $\subseteq Q \times X$ shows the set of initial states;

- $f: Q \times X \times U \times D \rightarrow T X$ and $h: Q \times X \rightarrow Y$ denote the vector fields which define the dynamics of the continuous and output states, and $T X$ represent the tangent space to $X$;

- $R: Q \times Q \times X \rightarrow X$ denote the continuous state resets.

Considering the electrified powertrain system, if we define the contact of the backlash as being positive when a half shaft is transmitting a driving torque, then the contact is negatively engaged when regenerative braking torque is transmitted via the haft shaft. Based on the above defined hybrid system, according to equations (1)-(7), the electric powertrain system can be described as:

- $Q=\left\{q_{1}, q_{2}, q_{3}\right\}$

- $X=\left\{T_{m}, \dot{\theta}_{m}, \dot{\theta}_{w}, \theta_{d}, \theta_{b}, T_{h s}\right\}$;

- $U=\left\{T_{m, r e f}, T_{h b}\right\}$;

- $D=\left\{T_{l}\right\}$

- $Y=\left\{T_{m}, \dot{\theta}_{m}, \dot{\theta}_{w}\right\}$.

As the hybrid automata shown in Fig. 5, the system is comprised of three discrete states, namely, $\mathrm{q}_{1}, \mathrm{q}_{2}$, and $\mathrm{q}_{3}$, indicating "contact negatively engaged", "backlash traversing" and "contact positively engaged", respectively. And each discrete state has its own dynamics for the evolution of the continuous state variables. The dynamics of the continuous state variables in each discrete state can be given by:

$$
\left\{\begin{array}{l}
\dot{x}(t)=A_{i} x(t)+B_{i} u(t)+F_{i} d(t) \\
y(t)=C_{i} x(t)
\end{array}\right.
$$

where $A_{i}, B_{i}, C_{i}$ and $F_{i}$ in each discrete state can be given by:

$$
A_{1}=A_{3}=\left(\begin{array}{cccccc}
-\frac{1}{\tau_{m}} & 0 & 0 & 0 & 0 & 0 \\
\frac{1}{J_{m}} & -\frac{b_{m}}{J_{m}} & 0 & 0 & 0 & -\frac{2}{i_{g} J_{m}} \\
0 & 0 & -\frac{b_{v}}{J_{v}} & 0 & 0 & \frac{2}{J_{v}} \\
0 & 1 / i_{g} & -1 & 0 & 0 & 0 \\
0 & 0 & 0 & 0 & 0 & 0 \\
\frac{c_{h s}}{i_{g} J_{m}} \frac{J_{m} k_{h s}-b_{m} c_{h s}}{i_{g} J_{m}} & \frac{b_{v} c_{h s}-J_{v} k_{h s}}{J_{v}} & 0 & 0 & -\left(\frac{2 c_{h s}}{i_{g}^{2} J_{m}}+\frac{2 c_{h s}}{J_{v}}\right)
\end{array}\right),
$$

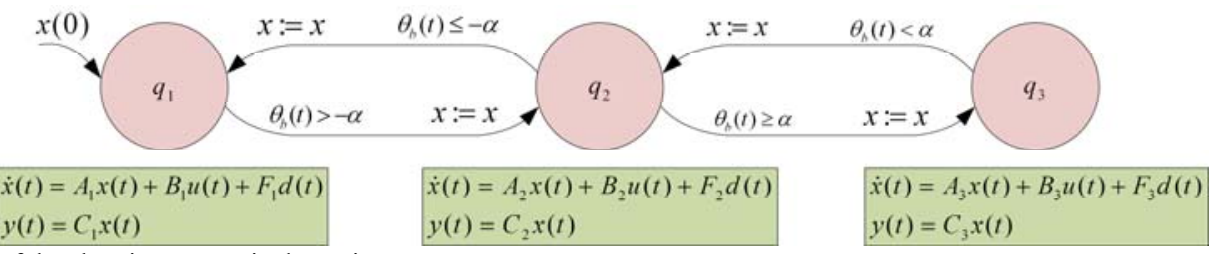

Fig. 5. Hybrid automata of the electric powertrain dynamics. 


$$
\begin{gathered}
A_{2}=\left(\begin{array}{cccccc}
-\frac{1}{\tau_{m}} & 0 & 0 & 0 & 0 & 0 \\
\frac{1}{J_{m}} & -\frac{b_{m}}{J_{m}} & 0 & 0 & 0 & 0 \\
0 & 0 & -\frac{b_{v}}{J_{v}} & 0 & 0 & 0 \\
0 & 1 / i_{g} & -1 & 0 & 0 & 0 \\
0 & 1 / i_{g} & -1 & \frac{k_{h s}}{c_{h s}} & -\frac{k_{h s}}{c_{h s}} & 0 \\
0 & 0 & 0 & 0 & 0 & 0
\end{array}\right) ; \\
B_{1}=B_{3}=\left(\begin{array}{cc}
\frac{1}{\tau_{m}} & 0 \\
0 & 0 \\
0 & -\frac{1}{J_{v}} \\
0 & 0 \\
0 & 0 \\
0 & \frac{c_{h s}}{J_{v}}
\end{array}\right) ; B_{2}=\left(\begin{array}{cc}
\frac{1}{\tau_{m}} & 0 \\
0 & 0 \\
0 & -\frac{1}{J_{v}} \\
0 & 0 \\
0 & 0 \\
0 & 0
\end{array}\right) ;
\end{gathered}
$$$$
C_{1}=C_{2}=C_{3}=\left(\begin{array}{llllll}
1 & 1 & 1 & 0 & 0 & 0
\end{array}\right)^{T} ; F_{1}=F_{3}=\left(\begin{array}{cccccc}
0 & 0 & -\frac{1}{J_{v}} & 0 & 0 & \frac{c_{h s}}{J_{v}}
\end{array}\right)^{T},
$$$$
F_{2}=\left(\begin{array}{llllll}
0 & 0 & -\frac{1}{J_{v}} & 0 & 0 & 0
\end{array}\right)^{T} \text {. }
$$

Fig. 6 shows the responses of the powertrain system to a sine wave torque input, significant oscillation of the half-shaft torque occurs because of the excitation of hard backlash nonlinearity and drivetrain flexibility. Focusing on the torque transition procedure, the operation mode of the electric powertrain is changed from drive to brake. As shown in the subplot of the figure, the backlash traverse happens approximately at $4 \mathrm{~s}$ and lasts for $190 \mathrm{~ms}$. In the backlash gap, the electric motor is decoupled from the load, i.e., the half-shaft torque $T_{h s}$ is zero, resulting in a temporary termination of the power transmission. Besides, when the contact is re-engaged again at the negative side, the contact impact causes great torque oscillations on the half-shaft, as the subplot illustrates, effecting the powertrain performance and vehicle drivability as well.

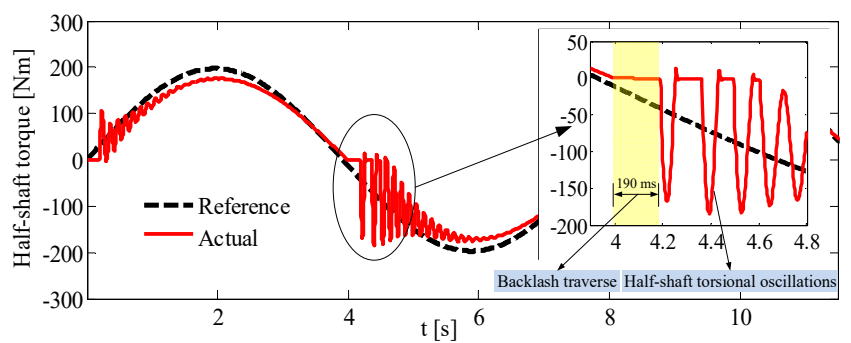

Fig. 6. Responses of the powertrain system to a sine wave torque input.

\section{HYBRID-STATE OBSERVER SYNTHESIS}

To further enhance the drivability of the electric vehicle during various state transitions, an active controller for powertrain backlash compensation needs to be developed. However, in the real vehicle application, the backlash position and half-shaft torque are unable to be directly measured by sensors on the plant. Therefore, the development of an observer for unmeasurable states' estimation is necessary. To cope with the hybrid physical plant, a hybrid architecture is selected for the observer.

Definition 2. [34] A hybrid-state observer $\mathcal{H}_{O}$ in a given hybrid system $\mathcal{H}$ such that

- receives the discrete-state output $\psi(k)$ of $\mathcal{H}$ as the discrete-state input, and the continuous-state input $u(t)$ and output $y(t)$ of $\mathcal{H}$ as the continuous-state inputs;

- provides $\tilde{q}(k)$ and $\tilde{x}(t)$ as the estimations of the current discrete and continuous states of $\mathcal{H}$, respectively;

\section{A. High-Level Architecture and Specification}

As shown in Fig. 7, the high-level framework of the proposed hybrid-state observer $\mathcal{O}_{d s c}$ contains two blocks:

1. A discrete-state observer $\mathcal{O}_{d s c}$ for the identification of the discrete state;

2. A continuous-state observer $\mathcal{G}_{c n t}$ for the estimation of the continuous states.

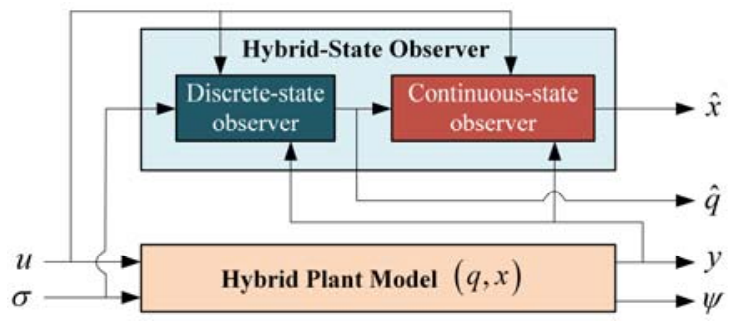

Fig. 7. High-level framework of the proposed hybrid-state observer.

The discrete-state observer $\mathcal{O}_{d s c}$ receives the continuousstate input $u(t)$ of the plant and measured output $(\psi(k), y(t))$ as multi-input, and provides the estimation $\tilde{q}(k)$ of the current discrete state of the hybrid system plant. The estimated discrete state is then utilized by the continuous-state observer for constructing the estimation of the system's continuous state variables $\tilde{x}(t)$. Besides, the continuous-state input $u(t)$ and output $y(t)$ of the hybrid system plant are also used by the continuous-state observer.

Based on the above structure, the specification for the proposed hybrid-state observer can be described as follows. Given constants $M>0, \lambda>0$ and $Z \geq 0$, the observation error of the hybrid-state observer is required to satisfy the exponentially bounded stability property [35].

$$
\|\zeta(t)\| \leq M\|\zeta(0)\| e^{-\lambda t}+Z \quad \forall t>0
$$

where $\zeta$ is the observation error, $\lambda$ denotes the convergence rate, and $Z$ represents the ultimate bound. 


\section{B. Discrete-State Observer Design for Backlash Estimation}

In this study, the discrete-state observer is required to identify the dynamics that the continuous state of the hybrid system plant is obeying to from a set of known ones. Assuming that the discrete-state observer has properly recognized that the hybrid system plant $\mathcal{H}_{p}$ is currently in the discrete state $q_{i}$, i.e. $\tilde{q}=q_{i}$, then the discrete-state observer should detect a deviation from the updates of $u(t)$ and $y(t)$ when the plant changes the discrete state to some $q_{j} \neq q_{i}$ and should also correctly identify the new discrete state $q_{i}$.

The time delay in the detection of the discrete-state transition is critical to the convergence of the whole hybrid-state observer. We denote the upper bound of such time delay by $\Delta$, and define a deviation signal $\delta$. When the hybrid system is in the discrete state of $q_{i}$, the $i$-th deviation generator produces a corresponding signal $\delta_{i}$, which should converge to zero. For designing such a deviation generator, in this study we adopt a Luenberger observer [36]. It can be established through the continuous-state dynamics associated to discrete state $q_{i}$ to be detected:

$$
\left\{\begin{array}{l}
\dot{\hat{x}}(t)=\left(A_{i}-L_{i} C_{i}\right) \hat{x}(t)+B_{i} u(t)+L_{i} y(t) \\
\delta_{i}(t)=C_{i} \hat{x}(t)-y(t)
\end{array}\right.
$$

where, $L_{i}$ is a design parameter indicating the converging rate of $\delta_{i}$, and $A_{i}, B_{i}$ and $C_{i}$ are the same ones of equations (10).

Then, the decision of the transition to $i$-th discrete state can be made based on the update of the deviation signal $\delta_{i}$. A simple approach for constructing the discrete-state transition indicator can be expressed by [37]:

$$
\Phi_{i}(t)= \begin{cases}1 & \text { if }\left\|\delta_{i}(t)\right\| \leq \sigma \\ 0 & \text { if }\left\|\delta_{i}(t)\right\|>\sigma\end{cases}
$$

where, the threshold $\sigma>0$ is a design parameter.

In the following proposition, a sufficient condition for ensuring $\Phi_{i}=$ true in a time $\Delta$ after a transition of the hybrid plant $\mathcal{H}_{p}$ to a dynamics $\left(A_{i}, B_{i}, C_{i}\right)$ is presented.

Proposition 1. Given $\Delta>0, \sigma>0$, and a upper bound $Z_{0}$ on $\|x-\hat{x}\|$, if the estimator gain matrix $L_{i}$ in equation (12) is chosen such that

$$
\alpha_{i}\left(A_{i}-L_{i} C_{i}\right) \leq-\frac{1}{\Delta} \log \frac{n\left\|C_{i}\right\| k\left(A_{i}-L_{i} C_{i}\right) Z_{0}}{\sigma}
$$

then $\Phi_{i}$ becomes true with a time period smaller than $\Delta$ after the plant dynamics parameters being switched to $\left(A_{i}, B_{i}, C_{i}\right)$.

\section{Proof of Proposition 1:}

Assume that the physical plant enters the discrete state $q_{i}$ at time $t_{0}$. Introduing the $j$-th deviation signal, and combining equation (12), then we can obtain

$$
\left[\begin{array}{c}
\dot{x} \\
\dot{x}-\dot{\hat{x}}_{j}
\end{array}\right]=\left[\begin{array}{cc}
A_{i} & 0 \\
\left(A_{j}-A_{i}\right)-L_{j}\left(C_{j}-C_{i}\right) & A_{j}-L_{j} C_{j}
\end{array}\right]\left[\begin{array}{c}
x \\
x-\hat{x}_{j}
\end{array}\right]+\left[\begin{array}{c}
B_{i} \\
B_{j}-B_{i}
\end{array}\right] u
$$

$$
\delta_{j}=\left[\begin{array}{ll}
C_{j}-C_{i} & C_{j}
\end{array}\right]\left[\begin{array}{c}
x \\
x-\hat{x}_{j}
\end{array}\right]
$$

For the dynamics of the continuous states associated with parameters of $\left(A_{i}, B_{i}, C_{i}\right)$ when $i=j$, deriving from the above representation of $\delta_{j}$, then we have

$$
\delta_{i}(t)=C_{i}\left(x\left(t_{0}\right)-\hat{x}_{i}\left(t_{0}\right)\right) e^{\left(A_{i}-L_{i} C_{i}\right)\left(t-t_{0}\right)}
$$

Let $A$ be a matrix in $\mathbb{R}^{n \times n}$. Denote the spectral abscissa and the condition number of matrix $A$ with respect to the matrix $T$ by $\alpha(A)$ and $k(A)=\|T\|\left\|T^{-1}\right\|$, respectively, such that $T^{-1} A T$ is in the Jordan canonical form. Then we have [38]:

$$
\left\|e^{A \tau}\right\| \leq n k(A) e^{\alpha(A) \tau} \quad \forall \tau \geq 0
$$

Thus, combining equation (17) and inequality (18),

$$
\begin{aligned}
\left|\delta_{i}(t)\right| & \leq\left\|C_{i}\right\|\left\|x(0)-\hat{x}_{i}(0)\right\|\left\|e^{\left(A_{i}-L_{i} C_{i}\right)\left(t-t_{0}\right)}\right\| \\
& \leq\left\|C_{i}\right\|\left\|x(0)-\hat{x}_{i}(0)\right\|\left\|n k\left(A_{i}-L_{i} C_{i}\right) e^{\alpha\left(A_{i}-L_{i} C_{i}\right)\left(t-t_{0}\right)}\right\| \\
& \leq\left\|C_{i}\right\| n k\left(A_{i}-L_{i} C_{i}\right) e^{\Delta \alpha\left(\mathbf{A}_{i}-L_{i} C_{i}\right)} Z_{0} \quad \forall t \geq t_{0}+\Delta
\end{aligned}
$$

which ensures $\left|\delta_{i}(t)\right|<\sigma$ for $t \geq t_{0}+\Delta$, by selecting the value of $L_{i}$ according to inequality (14).

Q.E.D.

Thus, the above transition indicator $\Phi_{i}$ detects the transitions of the hybrid system's discrete state between $q_{1}, q_{2}$ , and $q_{3}$, and is able to correctly identify the current discrete state of the hybrid system. The overall structure and identification processes of the above developed discrete-state observer is illustrated in Fig. 8.

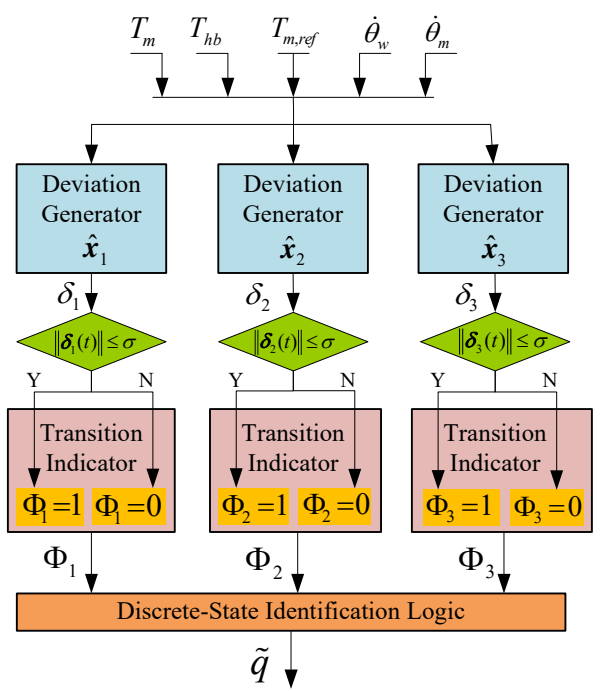

Fig. 8. Identification processes of the discrete-state observer.

\section{Continuous-State Observer Design for Half-Shaft Torque Estimation}

The continuous-state dynamics of the hybrid-state observer determines the estimation performance of the system's continuous states.

As shown in Fig. 9, in each identified discrete state $\tilde{q}_{k}$, the 
observation of the continuous states $\tilde{x}(t)$ is associated to the continuous-state dynamics of the hybrid system:

$$
\left\{\begin{array}{l}
\dot{\tilde{x}}(t)=\tilde{f}(\tilde{q}(k), \tilde{x}(t), u(t), y(t)) \\
\tilde{f}=\left(A_{i}-G_{i} C_{i}\right) \tilde{x}(t)+B_{i} u(t)+G_{i} y(t) \quad \text { if } \quad \tilde{q}(k)=\tilde{q}_{i}
\end{array}\right.
$$

where $A_{i}, B_{i}$ and $C_{i}$ are the same with the ones in equations (9)-(11), and the observer gain matrix $G_{i}$ is a design parameter which is used to tune the convergence velocity under each discrete state.

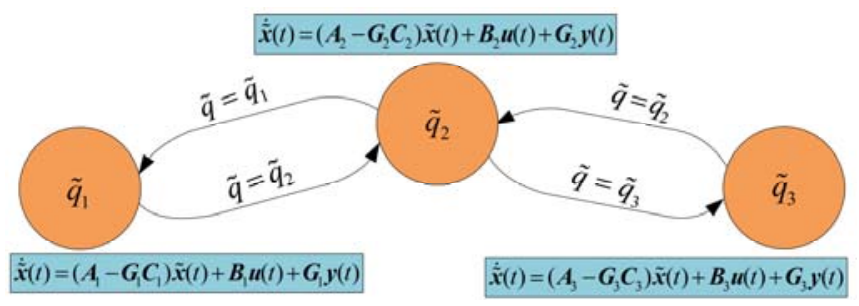

Fig. 9. Framework of the continuous-state observer.

Remark. Both discrete-state and continuous state observers provide estimation of continuous state $x(t)$. However, these two updates are separate. Because the discrete-state observer is expected to provide discrete-state identification with fast transients regardless of overshoots, while the continuous-state one is requested to generate a smooth estimation of the states with good robustness. This is the reason why both these two observers are essential and required.

\section{Convergence Analysis of the Hybrid-State OBSERVER}

The convergence properties of the proposed hybrid-state observer are investigated in this section. Since the physical plant has $N$ discrete-state states, and correspondingly the hybrid-state observer has $N$ discrete states as well. Thus the hybrid system, which is comprised of the physical plant and the hybrid-state observer, has $N \times N$ discrete states in total. Each of these states can be represented by $\left(q_{i}, \tilde{q}_{j}\right)$, where $q_{i}$ denotes the current real discrete state of the physical plant, and $\tilde{q}_{j}$ represents the current discrete state estimated by the hybridstate observer. For each $\left(q_{i}, \tilde{q}_{j}\right)$, the dynamics of the observation error $\zeta(t)=\tilde{x}(t)-x(t)$ can be represented by:

$$
\dot{\zeta}(t)=\left(A_{j}-G_{j} C_{j}\right) \zeta(t)+\left[\left(A_{i}-A_{j}\right)-G_{j}\left(C_{i}-C_{j}\right)\right] x(t)+\left(B_{i}-B_{j}\right) u(t)
$$

By integrating equation (21), then:

$$
\zeta(t)=e^{\left(A_{j}-G_{j} C_{j}\right) t} \zeta(0)+e^{\left(A_{j}-G_{j} C_{j}\right) t} * v(t)
$$

where $*$ represents the convolution operation, and $v(t)$ can be calculated by

$$
v(t)=\left[\left(A_{i}-A_{j}\right)-K\left(C_{i}-C_{j}\right)\right] x(t)+\left(B_{i}-B_{j}\right) u(t)
$$

For some $X>0$ and $U>0$, assume that $\|x(t)\|_{\infty} \leq X$ and $\|u(t)\|_{\infty} \leq U$, then we obtain [37]:

$$
\|v(t)\|_{\infty} \leq V=\left\|\left(A_{i}-A_{j}\right)-G_{j}\left(C_{i}-C_{j}\right)\right\|_{1} X+\left\|B_{i}-B_{j}\right\|_{1} U
$$

Consider a scenario that the hybrid plant $\mathcal{H}_{p}$ exhibits two subsequent transitions at time $t_{k}$ and $t_{k+1}$, respectively, and the discrete-state observer $\mathcal{O}_{d s c}$ identifies the above two state transitions at time $t_{k}^{\prime}$ and $t_{k+1}^{\prime}$, individually. Assume that the time interval between every two transitions is no less than $D>0$, and the discrete-state observer $\mathcal{O}_{d s c}$ identifies each transitions in the hybrid plant within a time period $\Delta<D$. Then we obtain

$$
\left\{\begin{array}{l}
t_{k+1}-t_{k} \geq D \\
t_{k}^{\prime}-t_{k} \leq \Delta \\
t_{k+1}^{\prime}-t_{k+1} \leq \Delta
\end{array}\right.
$$

In the time interval $\left[t_{k}^{\prime}, t_{k+1}\right], \tilde{q}_{i}=q_{i}$, thus, convergence of the observation error $\zeta(t)$ at a desired velocity can be achieved through a proper selection of the gain matrix $G_{i}$. However, in the time interval $\left[t_{k+1}, t_{k+1}^{\prime}\right], \tilde{q}_{i} \neq q_{j}$, the observation error $\zeta(t)$ is affected by the convergence in the previous time interval $\left[t_{k}^{\prime}, t_{k+1}\right]$ and may fail to converge. Therefore, the convergent behavior for time intervals $\left[t_{k}^{\prime}, t_{k+1}\right]$ and $\left[t_{k+1}, t_{k+1}^{\prime}\right]$ needs to be investigated, and compensation approach for ensuring the convergence of the observation error with a desired velocity during the whole time history is to be explored.

Based on equation (22), we have

$$
\begin{array}{cc}
\zeta(t)=e^{\left(A_{j}-G_{j} C_{j}\right)\left(t-t_{k}^{\prime}\right)} \zeta\left(t_{k}^{\prime}\right) \quad \forall t \in\left[t_{k}^{\prime}, t_{k+1}\right] \\
\zeta(t)=e^{\left(A_{j}-G_{j} C_{j}\right)\left(t-t_{k}^{\prime}\right)} \zeta\left(t_{k}^{\prime}\right) & \\
+\int_{0}^{t-t_{k+1}} e^{\left(A_{j}-G_{j} C_{j}\right)\left(t-t_{k+1}-\tau\right)} v\left(\tau+t_{k+1}\right) d \tau & \forall t \in\left(t_{k+1}, t_{k+1}^{\prime}\right]
\end{array}
$$

According to the inequality (18), for all $\left[t_{k}^{\prime}, t_{k+1}\right]$, the upper bound of the observation error $\zeta(t)$ can be given by

$$
\|\zeta(t)\| \leq n k\left(A_{j}-G_{j} C_{j}\right) e^{\alpha\left(A_{j}-G_{j} C_{j}\right)\left(t-t_{k}^{\prime}\right)}\left\|\zeta\left(t_{k}^{\prime}\right)\right\|
$$

It can be seen that the first term in equation (26-2) is the same with that of the equation (26-1). Therefore, it can also be bounded by the upper limit given by the inequality (27). And for the second term of the equation (26-2), considering inequality (24), then we have

$$
\begin{aligned}
& \left\|\int_{0}^{t-t_{k+1}} e^{\left(A_{j}-G_{j} C_{j}\right)\left(t-t_{k+1}-\tau\right)} v\left(\tau+t_{k+1}\right) d \tau\right\| \\
& \leq n k\left(A_{j}-G_{j} C_{j}\right) \int_{0}^{t-t_{k+1}} e^{\alpha\left(A_{j}-G_{j} C_{j}\right)\left(t-t_{k+1}-\tau\right)}\left\|v\left(\tau+t_{k+1}\right)\right\| d \tau \\
& \leq n k\left(A_{j}-G_{j} C_{j}\right)\|v(t)\|_{\infty} \int_{0}^{t-t_{k+1}} e^{\alpha\left(A_{j}-G_{j} C_{j}\right) \tau} d \tau \\
& =n k\left(A_{j}-G_{j} C_{j}\right) V \frac{e^{\alpha\left(A_{j}-G_{j} C_{j}\right)\left(t-t_{k+1}\right)}-1}{e^{\alpha\left(A_{j}-G_{j} C_{j}\right)}} \\
& \leq\left[n k\left(A_{j}-G_{j} C_{j}\right) V\right]\left(t-t_{k+1}\right)
\end{aligned}
$$

Then, combining (26-2), (27) and (28), for all $t \in\left(t_{k+1}, t_{k+1}^{\prime}\right]$, we have

$$
\|\zeta(t)\| \leq e^{\alpha\left(A_{j}-G_{j} C_{j}\right)\left(t-t_{k}^{\prime}\right)}\left\|\zeta\left(t_{k}^{\prime}\right)\right\|+\left[n k\left(A_{j}-G_{j} C_{j}\right) V\right]\left(t-t_{k+1}\right)
$$

Consider a worst case that the time interval between two state transitions of the hybrid system is exactly equal to $D$, and the 
discrete-state observer takes exact $\Delta$ to identify the transition in the plant. Thus, according to inequality (29), with $t=t_{k+1}^{\prime}$, the upper bound of the observation error $\zeta(t)$ for all $t \in\left(t_{k+1}, t_{k+1}^{\prime}\right]$ can be calculated by:

$$
\left\|\zeta\left(t_{k+1}^{\prime}\right)\right\| \leq e^{\alpha\left(A_{j}-G_{j} C_{j}\right) D}\left\|\zeta\left(t_{k}^{\prime}\right)\right\|+n k\left(A_{j}-G_{j} C_{j}\right) V \Delta
$$

In order to guarantee the good convergent performance of the hybrid-state observer in different time intervals, we put a strict requirement that $\left\|\zeta\left(t_{k+1}^{\prime}\right)\right\|=\left\|\zeta\left(t_{k}^{\prime}\right)\right\|$, i.e.

$$
e^{\alpha\left(A_{j}-G_{j} C_{j}\right) D}\left\|\zeta\left(t_{k}^{\prime}\right)\right\|+n k\left(A_{j}-G_{j} C_{j}\right) V \Delta=\left\|\zeta\left(t_{k}^{\prime}\right)\right\|
$$

then we can obtain

$$
\left\|\zeta\left(t_{k+1}^{\prime}\right)\right\| \leq \frac{n k\left(A_{j}-G_{j} C_{j}\right) V \Delta}{1-e^{\alpha\left(A_{j}-G_{j} C_{j}\right) D}}
$$

Besides, in order to reach a desired convergence velocity, we request the converge velocity to be greater than $\lambda>0$, which is shown as follows.

$$
\left\|\zeta\left(t_{k+1}^{\prime}\right)\right\| \leq e^{-\lambda D}\left\|\zeta\left(t_{k+1}^{\prime}\right)\right\|
$$

This above criterion can be achieved when $G_{j}$ is properly chosen according to

$$
e^{\alpha\left(A_{j}-G_{j} C_{j}\right) D}\left\|\zeta\left(t_{k}^{\prime}\right)\right\|+n k\left(A_{j}-G_{j} C_{j}\right) V \Delta \leq e^{-\lambda D}\left\|\zeta\left(t_{k+1}^{\prime}\right)\right\|
$$

Therefore, based on inequalities (32) and (33), we can conclude that the proposed observer is exponentially converges with velocity greater than $\lambda$. And this satisfies the exponentially bounded stability property required by the observer specification given by inequality (11).

$$
\|x(t)-\tilde{x}(t)\| \leq \frac{n k\left(A_{j}-G_{j} C_{j}\right) V \Delta}{1-e^{\alpha\left(A_{j}-G_{j} C_{j}\right) D}}
$$

\section{Hybrid-State ObSERVER VALidation AND RESUlts}

Performance and robustness are of great importance for cyber-physical systems [39-42]. In order to validate the feasibility and the effectiveness of the proposed hybrid-state observer on estimating system's continuous and discrete states, tests of the powertrain system with online simultaneous identification of the half-shaft torque and backlash position are carried out in this section.

\section{A. Validation Methodology and Scenario Design}

The dynamical models of the electrified powertrain with half-shaft flexibility and backlash nonlinearity, vehicle dynamics and the tyre, which are established in section 2 , are used to simulate the vehicle's operation. In the tests, the values of the vehicle states and the electrified powertrain system states generated by the models are regarded as the real values of the system. The inputs of the hybrid-state observer are comprised of those measurable states including electric motor's reference and real torques, master cylinder brake pressure, wheel cylinder brake pressure, wheel speed, motor speed, etc. These states are directly output from the models and passed to the hybrid-state observer. Finally, the feasibility and accuracy of the hybridstate observer estimation algorithm are verified by comparing the estimated half-shaft torque and the backlash position with the actual ones generated by the verified models.

Before the observer validation, the testing scenario is designed firstly as follows. As Fig. 10 shows, during testing the operation mode of the vehicle exhibits a transition from propulsion to regenerative deceleration. The initial speed of the vehicle is set at $40 \mathrm{~km} / \mathrm{h}$. During the first 3 seconds, the vehicle operates in its driving state with the output motor torque of $60 \mathrm{~N} \cdot \mathrm{m}$. In the $3 \mathrm{~s}$, the driver depresses the brake pedal, and the master cylinder brake pressure increases from 0 to $3 \mathrm{MPa}$, indicating the deceleration demand from driver. The electric motor responses rapidly, switching from the drive mode to the regenerative brake mode. Then, within the $3 \mathrm{~s}$, the motor speed presents torsional oscillations, which are caused by the backlash contact and half-shaft flexibility.
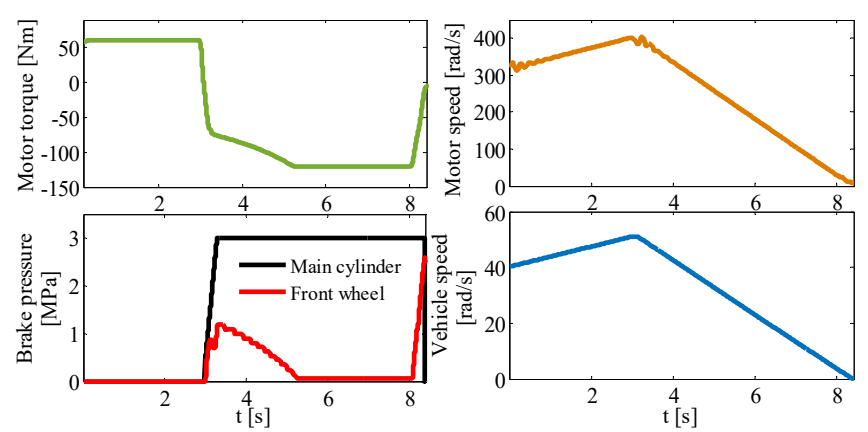

Fig. 10. Mode transition scenario of the vehicle for observer verification.

The aim of setting up the above test scenario is to activate the drivetrain nonlinearity, creating a harsh and sever environment that the operation mode rapidly switching, the backlash fiercely impacting and the half-shaft greatly vibrating. And then comprehensively evaluate the robustness and accuracy of the proposed hybrid-state observer under such a critical conditions.

\section{B. Validation Results and Discussion}

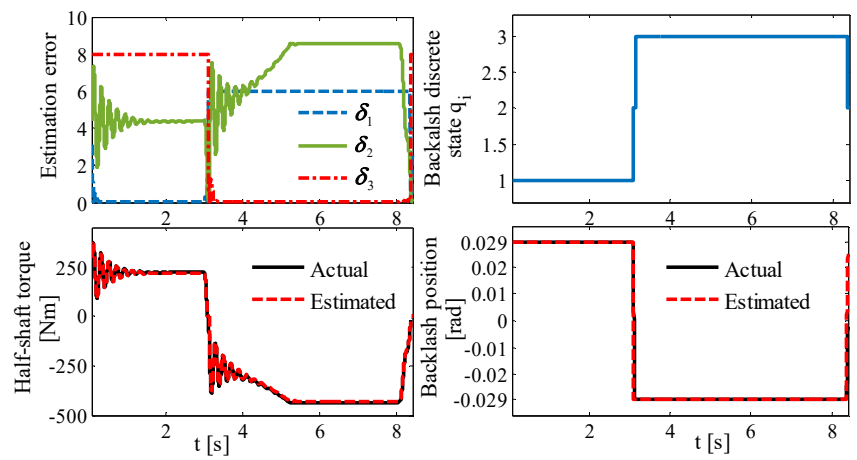

Fig. 11. Validation results of the hybrid-state observer under the testing scenario.

The plots of Fig. 11 shows the validation results of the hybrid-state observer under the established testing scenario. The upper-left plot illustrates the norm of the deviation signal $\delta_{i}(t)$ under the three different powertrain dynamics obtained via the discrete-state observer. Based on the above estimated deviation normal, the current discrete state of the backlash is then identified, as the upper-right plot shows. The last two plots of this figure illustrates the comparison of the estimated values 
of the half-shaft torque and backlash position as provided by the hybrid-state observer to the actual ones, respectively. According to on the validation results, the simultaneous estimated values of these two powertrain states precisely follow the real ones during the overall time history, demonstrating the robustness and effectiveness of the proposed hybrid-state observer.

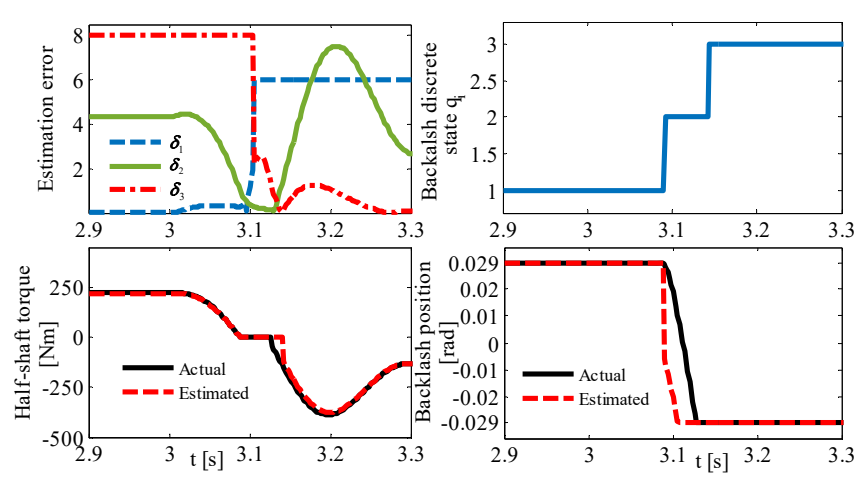

Fig. 12. Detailed results of the powertrain with the hybrid-state observer during state transition.

Fig. 12 represents the detailed situation of the operation state transition from propulsion to regenerative deceleration, which is happened around the $3 \mathrm{~s}$. The upper-right plot shows the variation of deviation norm $\left\|\delta_{i}\right\|$ of each discrete-state observer. Before $3.08 \mathrm{~s}$, the electrified powertrain works in the drive mode, and $\left\|\delta_{1}\right\|$ remains at a low level, while the other two norm signals are of high values. Thus, the current backlash state is identified as $q_{1}$, as shown in the upper-right plot, indicating the "positive contact". At around 3.08s, the system dynamics changes greatly with the operation mode switching to deceleration. Then the estimated deviation norm $\left\|\delta_{1}\right\|$ increases over the threshold with $\left\|\delta_{2}\right\|$ decreasing to below the bar. Correspondingly, the identified backlash state switches to $q_{2}$, i.e. the "backlash traverse". After the completion of the backlash traverse in the powertrain at around $3.14 \mathrm{~s}$, the norm $\left\|\delta_{2}\right\|$ rises again with norm signal $\left\|\delta_{3}\right\|$ being below the threshold. Hence, the discrete-state observer regulates its identification of the backlash position from $q_{2}$ to $q_{3}$, the "negative contact". Based on the dynamical variation of the observation of the discrete backlash state, the haft-shaft torque is precisely estimated in the meantime by the proposed continuous-state observer under each different discrete state. Besides, the figure clearly presents a small delay in detecting the change of the system dynamics during the powertrain mode transition, resulting in a tiny time delay in observing the halfshaft torque. According to the results, the estimation error of is within $2 \%$, and the convergence time is smaller than $15 \mathrm{~ms}$.

The above validation results demonstrates that the proposed hybrid-state observer can accurately and simultaneously estimate the half-shaft torque and backlash position of the electrified powertrain system under highly dynamical transition processes, verifying the feasibility, robustness and accuracy in harsh and complex situations.

\section{CONCLUSION}

As a typical cyber-physical system, advanced automotive mechatronic systems requires an increasing combination of mechanical, electrical/electronic, control and information disciplines. In this context, an algorithm dealing with simultaneous estimation of the backlash position and half-shaft torque for an automotive electric powertrain is proposed using a hybrid system approach in this paper.

System models, including the electric powertrain and vehicle dynamics models, were established with consideration of drivetrain backlash and flexibility. On the basis of the developed system models, the powertrain behavior was represented again using the hybrid automata according to the piecewise affine property of the backlash dynamics. Then, the hybrid-state observer, which is comprised of a discrete-state observer and a continuous-state observer, was designed for the simultaneous identification of the backlash position and halfshaft torque. The convergence property of the proposed hybridstate observer was investigated to guarantee the stability and reachability. Observer validation were carried out under a highly dynamical transition scenario of the vehicle states, and the results demonstrated the feasibility and effectiveness of the proposed hybrid-state observer.

Since social and human dynamic behaviors are considered to be an important and integral part of future CPS design and operation, further work can be carried out in the following areas: observation of Cyber-Physical-Social Systems (CPSS), exploration of different advanced estimation algorithms with the proposed high-level framework for hybrid-state observation, and design of compensatory torque controllers for improving powertrain performance based on the proposed hybrid-state observer.

\section{REFERENCES}

[1] Wang, F-Y. "The emergence of intelligent enterprises: From CPS to CPSS." IEEE Intelligent Systems 25, no. 4 (2010): 85-88.

[2] Li, H., Yan W., and Shi Y. "Continuous-time model predictive control of under-actuated spacecraft with bounded control torques." Automatica 75 (2017): 144-153.

[3] Wang, F-Y. "Control 5.0: from Newton to Merton in popper's cybersocial-physical spaces." IEEE/CAA Journal of Automatica Sinica 3, no. 3 (2016): 233-234.

[4] Gao, J., Proctor, A. A., Shi, Y., et ak. Hierarchical model predictive image-based visual servoing of underwater vehicles with adaptive neural network dynamic control. IEEE transactions on cybernetics 46 , no. 10 , (2016): 2323-2334.

[5] Martinez, C.M., Hu, X., Cao, D., Velenis, E., Gao, B. and Wellers, M. "Energy Management in Plug-in Hybrid Electric Vehicles: Recent Progress and a Connected Vehicles Perspective." IEEE Transactions on Vehicular Technology (2016), in press.

[6] Wang, F-Y. "From social computing to social manufacturing: the coming industrial revolution and new frontier in cyber-physical-social space." Bulletin of chinese Academy of Sciences 6 (2012): 001.

[7] Lv, C., Wang, H., and Cao, D. "High-Precision Hydraulic Pressure Control Based on Linear Pressure-Drop Modulation in Valve Critical Equilibrium State." IEEE Transactions on Industrial Electronics (2017).

[8] Cheng, X., Hu, X., Yang, L., Husain, I., Inoue, K., Krein, P., Lefevre, R., Li, Y., Nishi, H., Taiber, J.G. and Wang, F-Y. "Electrified vehicles and 
the smart grid: The ITS perspective." IEEE Transactions on Intelligent Transportation Systems 15, no. 4 (2014): 1388-1404..

[9] Tang, Y., Gao, H. and Kurths, J. "Robust Hळ Self-Triggered Control of Networked Systems Under Packet Dropouts." IEEE transactions on cybernetics 46, no. 12 (2016): 3294-3305.

[10] Hu, Y., Wang, F.Y. and Liu, X. "A CPSS approach for emergency evacuation in building fires." IEEE Intelligent Systems 29, no. 3 (2014) 48-52.

[11] Wang, L., Wang, Z., Huang, T. and Wei, G. "An event-triggered approach to state estimation for a class of complex networks with mixed time delays and nonlinearities." IEEE transactions on cybernetics 46, no. 11 (2016) 2497-2508.

[12] Yang, Chao, Liang Li, Sixiong You, Bingjie Yan, and Xian Du. "Cloud computing-based energy optimization control framework for plug-in hybrid electric bus." Energy 125 (2017): 11-26.

[13] Li, Z., Kolmanovsky, I.V., Atkins, E.M., Lu, J., Filev, D.P. and Bai, Y. "Road disturbance estimation and cloud-aided comfort-based route planning." IEEE transactions on cybernetics (2016), in press.

[14] Mathiyalagan, K., Su, H., Shi, P. "Exponential Hळ Filtering for DiscreteTime Switched Neural Networks With Random Delays." IEEE transactions on cybernetics 45, no. 4 (2015): 676-687.

[15] Lv, C., Zhang, J., Li, Y. and Yuan, Y. "Mode-switching-based active control of a powertrain system with non-linear backlash and flexibility for an electric vehicle during regenerative deceleration." Proceedings of the Institution of Mechanical Engineers, Part D: Journal of Automobile Engineering 229, no. 11 (2015): 1429-1442.

[16] Li, L., You, S., Yang, C., et al. "Driving-behavior-aware stochastic model predictive control for plug-in hybrid electric buses." Applied Energy 162 (2016): 868-879.

[17] Lagerberg, A. "Control and estimation of automotive powertrains with backlash." PhD diss., Chalmers University of Technology, 2004

[18] Bottiglione, F., Sorniotti, A., and Shead, L. "The effect of half-shaft torsion dynamics on the performance of a traction control system for electric vehicles." Proceedings of the Institution of Mechanical Engineers, Part D: Journal of Automobile Engineering 226, no. 9 (2012): 1145-1159.

[19] Lv, C., Zhang, J. and Li, Y. "Extended-Kalman-filter-based regenerative and friction blended braking control for electric vehicle equipped with axle motor considering damping and elastic properties of electric powertrain." Vehicle System Dynamics 52, no. 11 (2014): 1372-1388.

[20] Rostalski, P., Besselmann, T., Barić, M., Belzen, F.V. and Morari, M. "A hybrid approach to modelling, control and state estimation of mechanical systems with backlash." International Journal of Control 80, no. 11 (2007): 1729-1740.

[21] Stein, J.L. and Wang, C.H. "Estimation of gear backlash: Theory and simulation." Journal of dynamic systems, measurement, and control 120 , no. 1 (1998): 74-82.

[22] Ferrari-Trecate, G. and Gati, M. "Observability analysis and state observers for automotive powertrains with backlash: a hybrid system approach." International Journal of Control 79, no. 05 (2006): 496-507.

[23] Lagerberg, A. and Egardt, B. "Backlash estimation with application to automotive powertrains." IEEE Transactions on Control Systems Technology 15, no. 3 (2007): 483-493.

[24] Villwock, S. and Pacas, M. "Time-domain identification method for detecting mechanical backlash in electrical drives." IEEE Transactions on Industrial Electronics 56, no. 2 (2009): 568-573.

[25] Hedrick, J. K. "Estimation of Vehicle Shaft Torque Using Nonlinear Observers1." Journal of dynamic systems, measurement, and control 114 (1992): 395.

[26] Gao, B., Chen, H., Ma, Y. and Sanada, K. "Design of nonlinear shaft torque observer for trucks with Automated Manual Transmission." Mechatronics 21, no. 6 (2011): 1034-1042.

[27] Lv, C., Zhang, J., Li, Y. and Yuan, Y. "Directional-stability-aware brake blending control synthesis for over-actuated electric vehicles during straight-line deceleration." Mechatronics 38 (2016): 121-131.

[28] Krishnan, R. Electric motor drives: modeling, analysis, and control. Prentice Hall, 2001.

[29] Lagerberg, A. and Egardt, B. "Estimation of Backlash with Application to Automotive Powertrains." In Proceedings of the 42nd IEEE Conference on Decision and Control, Maui, Hawaii. 2003.

[30] Zhang, J., Lv, C., Gou, J. and Kong, D. "Cooperative control of regenerative braking and hydraulic braking of an electrified passenger car." Proceedings of the Institution of Mechanical Engineers, Part D Journal of Automobile Engineering 226, no. 10 (2012): 1289-1302.
[31] Lagerberg, A. and Egardt, B. "Model Predictive Control of Automotive Powertrains with Backlash." In 16th IFAC World Congress, Prague, Czech Republic. 2005.

[32] Pacejka, H. B., and I. J. M. Besselink. "Magic formula tyre model with transient properties." Vehicle system dynamics 27.S1 (1997): 234-249.

[33] Lygeros, J., Sastry, S. and Tomlin, C. "Hybrid Systems: Foundations, advanced topics and applications." (2012).

[34] Balluchi, A., Benvenuti, L., Di Benedetto, M.D. and SangiovanniVincentelli, A. "The Design of dynamical observers for hybrid systems: Theory and Application to an Automotive Control Problem." Automatica 49, no. 4 (2013): 915-925.

[35] Balluchi, A., Benvenuti, L., Benedetto, M.D.D. and SangiovanniVincentelli, A.. "Design of Observers for Hybrid Systems." In Proceedings of the 5th International Workshop on Hybrid Systems: Computation and Control, pp. 76-89. Springer-Verlag, 2002.

[36] Luenberger, D. "An introduction to observers." IEEE Transactions on automatic control 16, no. 6 (1971): 596-602.

[37] Balluchi, A., Benvenuti, L., Di Benedetto, M.D., and SangiovanniVincentelli, A. The design of dynamical observers for hybrid systems: Theory and application to an automotive control problem. Technical report, University of Rome, Dpt. Computer and System Sciences, 2012.

[38] Van Loan, C. "The sensitivity of the matrix exponential." SIAM Journal on Numerical Analysis 14, no. 6 (1977): 971-981.

[39] Lv, C., Zhang, J., Li, Y., Yuan, Y. "Novel control algorithm of braking energy regeneration system for an electric vehicle during safety-critical driving maneuvers." Energy conversion and management 106 (2015): 520-529.

[40] Wang, F-Y., Wang, X., Li, L., et al. "Steps toward Parallel Intelligence." IEEE/CAA Journal of Automatica Sinica 3, no. 4 (2016): 345-348.

[41] Lv, C., Zhang, J., Li, Y., Yuan, Y. "Mechanism analysis and evaluation methodology of regenerative braking contribution to energy efficiency improvement of electrified vehicles." Energy Conversion and Management 92 (2015): 469-482.

[42] Wang, F-Y., Zhang, J., Wei, Q, et al. "PDP: parallel dynamic programming." IEEE/CAA Journal of Automatica Sinica 4, no. 1 (2017): $1-5$. 
2017-08-22

\title{
Simultaneous observation of hybrid
}

states for cyber-physical systems: a

case study of electric vehicle powertrain

\author{
Lv, Chen
}

IEEE

Chen Lv, et al. Simultaneous observation of hybrid states for cyber-physical systems: a case study of electric vehicle powertrain, IEEE Transactions on Cybernetics, Volume 48, Issue 8, August 2018, pp2357-2367

http://dx.doi.org/10.1109/TCYB.2017.2738003

Downloaded from Cranfield Library Services E-Repository 